\title{
AlfaBtiCs para la mejora de habilidades y conocimientos digitales
}

\author{
Jesús Carlos Salazar Peña
}

\begin{abstract}
Resumen
Una de las principales problemáticas al introducir tecnologías en la educación, es la capacitación de los profesores, ya que sus procesos de adaptación han sido complicados pues incluso aquellos que ya han tenido un acercamiento con ellas, aún les resulta difícil su comprensión, aplicación, e implementación dentro las aulas. A pesar de los esfuerzos por realizar campañas de capacitación y de equipar las aulas con tecnología con pizarras electrónicas, proyectores, material digital, entre otros; aún existen aulas en las cuales dicho material se ha vuelto obsoleto, por una mala administración y falta de interés por parte del cuerpo educativo.

La propuesta que presentamos a la problemática antes mencionada es la elaboración de un curso digital, multimedia e interactivo al que llamamos AlfaBtiCs, que a manera de alfabetización digital permite a los profesores comprender de mejor manera algunos de los elementos básicos tales como la interfaz de usuario, la navegación por internet y la búsqueda, uso y presentación de información.
\end{abstract}

Palabras clave: alfabetización digital, medios digitales, capacitación, conocimiento.

\section{AlfaBtiCs for the improvement of the digital SKILls and KNOWLedge}

\begin{abstract}
One of the main problems when introducing technologies in education is the training of teachers, since their adaptation processes have been complicated because even those who have already had an approach with them, still find it difficult to understand, apply, and implementation within classrooms. Despite the efforts to carry out training campaigns and equip classrooms with technology with electronic boards, projectors, digital material, among others; there are still classrooms in which such material has become obsolete, due to poor administration and lack of interest on the part of the educational body.

The proposal we present to the afore mentioned problem is the development of a digital, multimedia and interactive course that we call AlfaBtiCs, as a digital literacy allows teachers to better understand some of the basic elements of the different media, such as user interface, internet browsing, search, use and presentation of information.
\end{abstract}

Keywords: digital literacy, digital media, training, knowledge.

Recepción: 02/03/2020. Aprobación: 18/01/2021. Dol: http://doi.org/10.22201/cuaieed.16076079e.2021.22.2.7 
"AlfaBtiCs para la mejora de habilidades y conocimientos digitales" Jesús Carlos Salazar Peña

Vol. 22, Núm. 2, marzo-abril 2021 Revista Digital Universitaria

\section{Jesús Carlos Salazar Peña}

jesuscsalazar88@gmail.com

orcid.org/0000-0003-1670-7695

Obtuvo la licenciatura en Informática Administrativa en el año 2010, ese mismo año participó en el programa Delfín de CONACYT para realizar una estancia de investigación en la Universidad de Murcia, España con el Dr. Joaquin Nicolas Ros, posteriormente ingresó a los servicios de educación pública como profesor de educación secundaria. En el año 2015 finalizó la Maestría en educación en la Universidad Autónoma de Nayarit la cual se encuentra en el Programa Nacional de Posgrados de Calidad (PNCP), durante este proceso realizó una estancia de investigación en Sevilla, España, con el Dr. Julio Cabero Almenara, del secretariado de Recursos Audiovisuales y nuevas tecnologías, tuve la participación en la creación de la revista universitaria meducLink, en colaboración con compañeros maestrantes (cabe destacar que el registro no se realizó) en donde se publicaron 3 números, además de la participación en el xı Foro Internacional sobre Evaluación de la Calidad de la Educación Superior y de la Investigación (FECIES) en Sevilla, España.

En el año 2017 comenzó el doctorado en educación en la Universidad Autónoma de Madrid, España, la cual finalizó en noviembre del año 2020, durante este proceso realizó una estancia de investigación en el Instituto de Investigación sobre la Universidad y la Educación, IISUE, con la Dra. Janeth TrejoQuintana, además de participaciones en el । Congreso Internacional de Formación de Profesorado e Innovación Educativa y en las iv Jornadas de Doctorado en Educación de la Universidad Autónoma de Madrid. 


\section{Introducción}

La presencia de las tecnologías, en el día a día, ha impuesto nuevas formas de pensar, de actuar y de aprender en la sociedad; las nuevas generaciones crecen en una cultura en donde impera el uso de dispositivos electrónicos con acceso a internet y a una infinidad de medios digitales y a bancos de información en diversos medios que les permiten hacer consultas en cualquier lugar y momento.

Hoy en día, podemos hacer casi todo con un dispositivo móvil con conexión a Internet, sin embargo, en algunas escuelas, la situación es diferente, ya que sus métodos de enseñanza existentes no están empatados con estas nuevas maneras de resolver situaciones, y todavía más, algunos profesores, de mayor antigüedad, han tenido una inadecuada inclusión en los medios digitales dentro de su práctica docente.

Para contextualizar, el término multimedia se acuñó ya en los años 80 a través de trabajos de Negroponte, Papert y Minsky en la Universidad de Massachusetts (міт). Las tecnologías de vanguardia fueron desarrollándose y generaron sistemas hipermedia capaces de trabajar con sonidos, gráficos, animación, videos y texto que permitieron la interactividad y la personalización, como consecuencia dieron origen a lo que hoy llamamos multimedia (Razquin, 1997).

Con la aparición de los multimedia, se hizo necesario el desarrollo de nuevas habilidades en los profesores para llevar a cabo su práctica educativa, pues las generaciones recientes de estudiantes ya no aprenden de la misma manera; los métodos de repetición y memorización se han vuelto obsoletos con la llegada de métodos de búsqueda, interpretación y presentación de la información. Hay muchos ejemplos de prácticas docentes alejadas del enciclopedismo, como el aprendizaje de las artes y la literatura jugando Beowulf (De Paula et al., 2018) o las clases de física en un entorno 3D combinado con realidad virtual (Bogusevschi et al., 2020).

Actualmente, el profesor tiene un papel de acompañamiento en el proceso de aprendizaje del estudiante, su función es la de guía, para que el estudiante logre por sí mismo generar nuevos conocimientos a partir de la consulta de los bancos de información que se encuentra en Internet, teniendo en cuenta sus características individuales, como la motivación, la autoeficacia o sus emociones (Schweder y Raufelder, 2019).

Rosen, D. (2020), menciona que las habilidades digitales básicas deben incluirse dentro de la educación para la alfabetización de adultos, ya que necesitan de una alfabetización digital para resolver los nuevos problemas que enfrentamos en la sociedad, incluidos los creados por la tecnología. 


\section{Desarrollo}

Se han elaborado algunos estándares orientadores para el análisis y desarrollo de nuevas habilidades en los profesores, tal es el caso de la unesco (2007), que en su documento: Competencias y estándares Tic desde la dimensión pedagógica: una perspectiva desde los niveles de apropiación de las tic en la práctica educativa docente, describen la formación de calidad que un docente debe tener en la actualidad para enfrentar el desafío de enseñar en una sociedad de la información, teniendo en cuenta los modelos actuales de gestión del conocimiento en la formación (Acevedo-Correa et al., 2020).

Otro documento, también desarrollado por la unesco (2011), es el Modelo de competencias de Alfabetización Mediática e Informacional (AMI). El objetivo de este proyecto cST es proporcionar una guía sobre cómo mejorar el desempeño de los profesores a través de las tic y dar una nueva dimensión a sus destrezas, sin importar en qué lugar esté ubicada el aula, lo que daría como resultado una mejor educación y unos estudiantes con mayores habilidades.

En México, por ejemplo, se puso en marcha el proyecto HDT (Habilidades Digitales para Todos) que se contempló en el Plan Nacional de Desarrollo 2009-2011 y que entró en vigor el primero de enero de 2009. Dicho programa consistió en el equipamiento de aulas multimedia con pizarras electrónicas y computadoras personales con conexión 1 a 30, es decir, el profesor podía gestionar los trabajos de hasta 30 estudiantes durante la sesión de clase; su objetivo general era "contribuir a mejorar el aprendizaje de los estudiantes de educación básica propiciando el manejo de tic en el sistema educativo mediante el acceso a las aulas telemáticas". (SEP, 2009). Este proyecto incluía también Objetos de Aprendizaje (ODA), los cuales estaban relacionados con los contenidos del currículum formal y se encontraban a disposición de los docentes para usarlos en las clases.

A pesar de estos esfuerzos, aún existen profesores con mayor antigüedad que no tienen una adaptación adecuada al uso de las tecnologías dentro de su práctica docente, a diferencia las personas que nacieron en una era donde el uso de las tecnologías es más común, lo que les permitió desarrollar y adaptar un "lenguaje" más cercano a los medios digitales. Hay muchos autores que describen las consecuencias de esta brecha digital causada, en parte, por la edad como por ejemplo, la investigadora Louise Starkey, de la Universidad de Victoria en Wellington, Nueva Zelanda, hace una amplia revisión de estos trabajos (Starkey, 2020).

El concepto de alfabetización ha sufrido algunas modificaciones debido a la aparición de nuevos lenguajes. Se habla que, anteriormente, una persona alfabeta era la que tenía la capacidad de leer y escribir, sin embargo, las demandas actuales sobre el lenguaje van más allá de solo la comprensión y uso del alfabeto, esto aunado a que las tecnologías han revolucionado la forma de comunicación 
"AlfaBtiCs para la mejora de habilidades y conocimientos digitales" Jesús Carlos Salazar Peña

Vol. 22, Núm. 2, marzo-abril 2021

Revista Digital Universitaria

pues permiten desenvolvernos en lenguajes donde se utilizan imágenes, sonidos, videos, entre otros elementos multimedia; podemos decir que, en el presente, existe una comunicación entre la computadora y el usuario en donde se tienen como intermediaria una interfaz integrada por símbolos, hipertextos, hipervínculos, botones con acciones específicas, entre otros. En consecuencia, es necesario, que las nuevas alfabetizaciones vayan encaminadas a comprender y manipular este tipo de comunicación visual e interactiva con el mundo digital.

Entonces, entendemos que hoy en día nos comunicamos mediante un lenguaje de medios, pues todo lenguaje es necesariamente un sistema que engloba y traduce otros lenguajes previos, dicho sistema traductor constituye su núcleo esencial, en un sistema de transformaciones. (Lotman, 1998).

Buckingham, D. (2006) menciona que "los educadores para los medios tienen que cuestionar el uso meramente instrumental de la tecnología y la idea de que la tecnología es simplemente una herramienta neutral para llevar información. Por el contrario, tenemos que definir y promover nuevas formas de 'alfabetización digital', extendiendo y quizás reconsiderando nuestros conocidos enfoques críticos en relación con los nuevos medios, como los juegos de computadora e Internet".

Eva Aladro (2017), en su documento: El lenguaje digital, una gramática generativa, hace una comparación de los usos y funciones del lenguaje digital con la teoría Chomskiana sobre la gramática transformacional y generativa, esta teoría se basa en que existe un lenguaje universal asociado a las profundas bases semánticas que consideramos innatas, que pueden ser adquiridas externamente, y que constituyen el fondo de recursos sobre el que se arma.

A partir de esta teoría, podemos decir que la falta de capacidad de los docentes que no tuvieron una formación en el uso de las tecnologías, se debe a que no gozaron de un acercamiento adecuado o "cotidiano" a las mismas, es decir, el cerebro de los llamados inmigrantes digitales, creó diferentes canales de aprendizaje para el uso de las tecnologías que estaban a su alcance durante su proceso de adaptación, sin embargo, y como se ha mencionado en diversas investigaciones, las tecnologías han evolucionado de manera rápida en comparación con la capacidad de adaptación de los docentes con mayor antigüedad; esto repercute de manera negativa en la comprensión e interacción con las nuevas interfaces; es decir, cada vez surgen más aplicaciones y más funciones al momento de utilizar las plataformas, blogs o programas educativos para el apoyo docente.

Por ello, se desarrolló e implementó el curso digital interactivo AlfaBtics, con la intención de proporcionar a los profesores una alternativa de capacitación que, por su carácter digital, pueden consultar en el momento lo que deseen y puedan resolver dudas sobre conceptos o procedimientos en el uso de los medios digitales. 
Imagen 1. Pantalla de inicio del curso AlfaBtiCs.

Imagen 2. Índice de contenidos del curso AlfaBtics.
El diseño de la interfaz del curso se realizó pensando en la facilidad y funcionalidad de este, es decir, se realizó un diseño simple e intuitivo en donde el profesor puede navegar entre sus diferentes apartados de manera sencilla.

A.m.e.c.

\section{Curso de} Alfabetización Digital

\section{ENIRAR}

También se decidió utilizar colores básicos y sencillos en cada uno de los módulos, con la intención de que el usuario pueda identificar en qué apartado se encuentra al momento de realizar el curso.

El curso AlfaBtics está formado por 3 módulos:

1. La interfaz de usuario.

2. El explorador de internet.

3. Acceso y uso de la información.

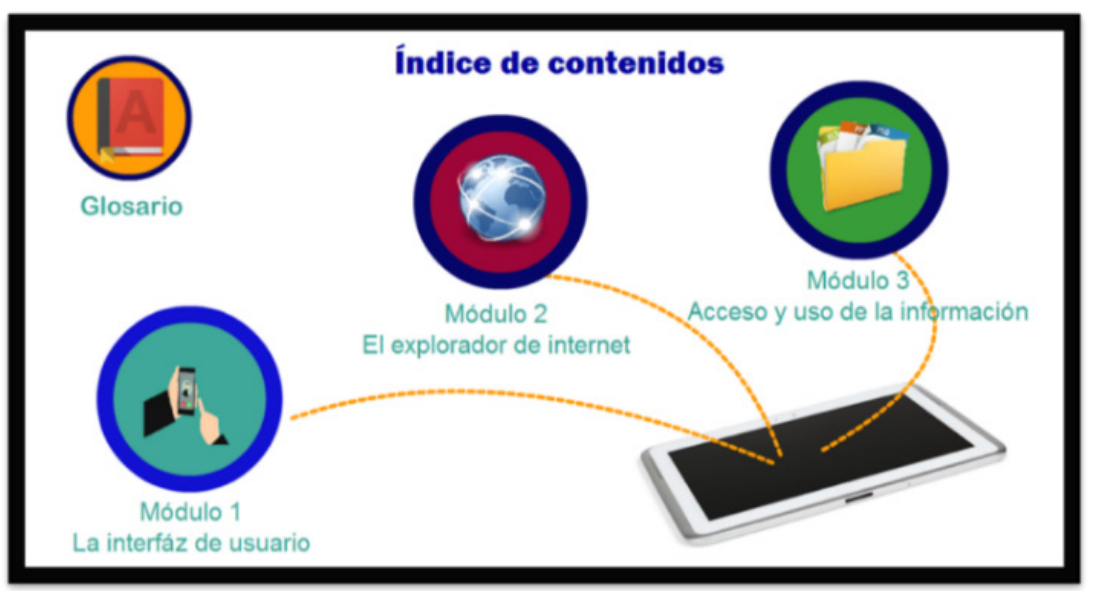

La interfaz fue diseñada para que el usuario pueda identificar sus elementos de manera sencilla. Estudios previos indican que uno de los factores que influye en el bajo compromiso de las personas son las interfaces, por lo que su buen diseño puede facilitar la comunicación entre los usuarios y la plataforma y además puede conducir a una mejor experiencia del usuario, aumentando así la satisfacción de los alumnos (Liu.S. et al., 2020). 
Imagen 3. Pantalla de contenido del curso AlfaBtiCs.
Cuadro 1. Resultados del primer y segundo cuestionario del apartado de los conocimientos.

Cuadro 2. Resultados del primer y segundo cuestionario del apartado de las habilidades.

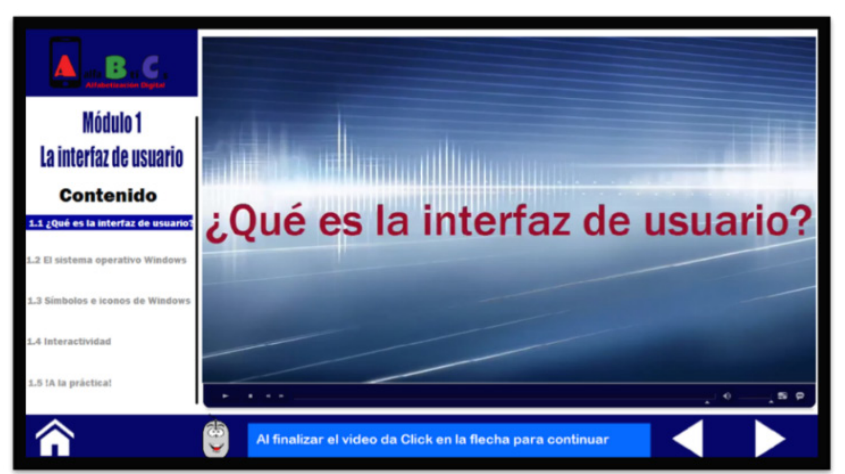

Otra de las finalidades del curso, es conocer el estado de los conocimientos y habilidades digitales de los profesores y determinar si el uso de este tipo de herramientas ayuda a incrementarlos o a mejorarlos; para ello, el curso se implementó a un total de 59 profesores de educación secundaria de la ciudad de Tepic en el estado de Nayarit, México.

Para recabar la información, se realizó un cuestionario de 40 ítems con el uso de la herramienta GoogleDocs, el cual se presentó en una página web de Google Sites que recabó información sociológica (5 ítems), sobre capacitación y uso de los medios digitales en el aula (5 ítems), sobre los conocimientos (15 ítems) y habilidades (15 ítems). El cuestionario se aplicó en dos momentos, el primero antes de realizar el curso y el segundo al finalizarlo (pre test, post test), los apartados de habilidades y conocimientos se realizaron mediante ítems con escala de Likert de 1 a 4, en donde 1 era nada y 4 era mucho. Los ítems están redactados de tal manera que el usuario pueda determinar y medir su autopercepción una vez realizado el curso AlfaBtiCs.

Con ayuda del software spss, se obtuvieron los promedios del primer y segundo cuestionario de los apartados de conocimientos y habilidades en donde se recabaron los siguientes resultados:

Sobre los conocimientos:

\begin{tabular}{|l|c|c|c|c|}
\hline \multicolumn{5}{|c|}{ Estadísticos descriptivos } \\
\hline & N & Mínimo & Máximo & Media \\
\hline Promedio primer cuestionario & 59 & 1.0 & 4.0 & 2.502 \\
Promedio segundo cuestionario & 59 & 1.9 & 4.0 & 3.473 \\
N válido (según lista) & 59 & & & \\
\hline
\end{tabular}

Sobre las habilidades:

\begin{tabular}{|l|c|c|c|c|}
\hline \multicolumn{5}{|c|}{ Estadísticos descriptivos } \\
\hline & N & Mínimo & Máximo & Media \\
\hline Promedio primer cuestionario & 59 & 1.0 & 4.0 & 2.511 \\
Promedio segundo cuestionario & 59 & 2.0 & 4.0 & 3.451 \\
N válido (según lista) & 59 & & & \\
\hline
\end{tabular}




\section{Conclusiones}

En los cuadros 1 y 2 se observan los resultados de los promedios obtenidos en los apartados de conocimientos y habilidades de los 59 profesores que participaron en el curso AlfaBtiCs. La información indica que en cada apartado hubo un incremento en la percepción que tienen los profesores acerca de sus conocimientos y habilidades en la alfabetización digital. Para visualizar mejor estos datos, presentamos la siguiente tabla:

\begin{tabular}{|l|c|c|c|c}
\hline \multicolumn{1}{|c|}{ Ppartados } & $\begin{array}{c}1^{\circ} \text { Cuestionario } \\
\text { (pre) }\end{array}$ & $\begin{array}{c}2^{\circ} \text { Cuestionario } \\
(\text { post) }\end{array}$ & & Incremento \\
Conocimientos & 2.502 & 3.473 & .971 & $27.95 \%$ \\
Habilidades & 2.511 & 3.451 & .940 & $27.23 \%$
\end{tabular}

Se observa un aumento de más del 25\% en la percepción que tienen los profesores sobre sus conocimientos y habilidades al finalizar el curso AlfaBtiCs, por lo que podemos concluir que este tipo de herramientas, diseñadas adecuadamente, pueden ser una alternativa dentro de las capacitaciones sobre alfabetización digital y el uso de los medios digitales para profesores de educación secundaria, incluso se pueden diseñar este tipo de cursos para mejorar conocimientos de cualquier tema y para cualquier segmento de la población.

\section{Referencias}

* Acevedo-Correa, Y., Aristizábal-Botero, C.A., Valencia-Arias, A., y Bran-Piedrahita, Lemy. (2020). Formulación de modelos de gestión del conocimiento aplicados al contexto de instituciones de educación superior. Información tecnológica, 31(1), 103-112. https://dx.doi.org/10.4067/S0718-07642020000100103

* Aladro, E. (2017). El lenguaje digital, una gramática generativa. cic. Cuadernos de Información y Comunicación, 22, 79-94. https://doi.org/10.5209/CIYC.55968

* Bogusevschi, D., Muntean, C. y Muntean, G.M. (2020). Teaching and Learning Physics using 3D Virtual Learning Environment: A Case Study of Combined Virtual Reality and Virtual Laboratory in Secondary School. Journal of Computers in Mathematics and Science Teaching, 39(1), 5-18. Waynesville, NC USA: Association for the Advancement of Computing in Education (AACE). Consultado el 05/08/2020 https://www.learntechlib.org/primary/p/210965/

* Buckingham, D. (2006). La educación para los medios en la era de la tecnología digital. Congreso del décimo aniversario de MED "La sapienza di comunicare".

- De Paula, B. H., Burn, A., Noss, R., y Valente, J. A. (2018). Playing Beowulf: Bridging computational thinking, arts and literature through game-making. International Journal of Child-Computer Interaction, 16, 39-46. https://doi.org/10.1016/j. ijcci.2017.11.003 
* Liu, S., Liang, T., Shao, S. y J. Kong. (2020). Evaluating Localized MOOCs: The Role of Culture on Interface Design and User Experience. IEEE Access, 8, 107927-107940. https://ieeexplore.ieee.org/stamp/stamp.jsp?arnumber=9057634

* Lotman, J. (1998). La semiosfera. La semiótica de la cultura. Cátedra. https://www. redalyc.org/pdf/105/10503908.pdf

* Organización de las Naciones Unidas para la Educación, la Ciencia y la Cultura (UNESCO). (2007). Normas UNESCO sobre Competencias en TIC para Docentes. Directrices para la aplicación.

* Organización de las Naciones Unidas para la Educación, la Ciencia y la Cultura (UNESCO). (2011). Alfabetización mediática e información. Curriculum para profesores.

* Razquín, P. (1997). Del hipertexto al multimedia interactivo. Evolución, situación actual y perspectivas de los sistemas y aplicaciones multimedia. Revista General de Información y Documentación, 7 (1), 107-132. https://revistas.ucm.es/index.php/ RGID/article/view/RGID9797120107A

* Rosen, D. (2020). Assessing and Teaching Adult Learners' Basic and Advanced 21st Century Digital Literacy Skills. Adult Literacy Education, 2(1), 73-75.

* Schweder, S. y Raufelder, D. (2019) Positive emotions, learning behavior and teacher support in self-directed learning during adolescence: Do age and gender matter? Journal of Adolescence, 73 (2019), pp. $73-84$ https://doi.org/10.1016/j. adolescence.2019.04.004

* Secretaria de Educación Pública (seP). (2009). Programa Habilidades digitales para Todos(HDT), Libro Blanco. https://sep.gob.mx/work/models/sep1/Resource/2959/5/ images/LB\%20HDT.pdf

- Starkey, L. (2020) A review of research exploring teacher preparation for the digital age, Cambridge Journal of Education, 50 (1,) 37-56. https://doi.org/10.1080/030576 4X.2019.1625867

\section{Cómo CITAR ESTE ARTículo}

* Salazar Peña, Jesús Carlos. (2021, marzo-abril). AlfaBtiCs para la mejora de habilidades y conocimientos digitales. Revista Digital Universitaria (RDU), 22(2). DOI: http://doi.org/10.22201/cuaieed.16076079e.2021.22.2.7 